\title{
On the system of two all_different predicates
}

\author{
G. Appa ${ }^{1}$, D. Magos ${ }^{2}$, I. Mourtos ${ }^{1}$ \\ ${ }^{1}$ London School of Economics and Political Science \\ Houghton Street, London WC2A 2AE \\ ${ }^{2}$ Technological Educational Institute of Athens \\ Ag. Spyridonos Str., Egaleo 122 10, Greece
}

Working Paper No: LSEOR 05.74

ISBN: 0753016966 
First published in Great Britain in 2005

by the Department of Operational Research

London School of Economics and Political Science

Copyright (C) The London School of Economics and Political Science, 2005

The contributors have asserted their moral rights.

All rights reserved. No part of this publication may be reproduced, stored in a retrieval system, or transmitted in any form or by any means, without the prior permission in writing of the publisher, nor be circulated in any form of binding or cover other than that in which it is published.

Typeset, printed and bound by:

The London School of Economics and Political Science

Houghton Street

London WC2A 2AE 


\title{
On the system of two all_different predicates
}

\author{
G. Appa ${ }^{1}$, D. Magos ${ }^{2, *}$, I. Mourtos ${ }^{1}$ \\ ${ }^{1}$ London School of Economics, London WC2A 2AE, UK. \\ email:\{g.appa,j.mourtos\}@lse.ac.uk \\ ${ }^{2}$ Technological Educational Institute of Athens, Ag. Spyridonos Str., Egaleo 122 10, Greece. \\ email:dmagos@teiath.gr
}

Keywords: combinatorial problems, all_different predicate, constraint logic programming, facet

\section{Introduction}

Numerous real-life problems require certain variables to be assigned different values. This requirement is encapsulated in constraints of difference. If $x_{1}, x_{2}$ denote two problem variables, the (nonlinear) constraint of difference is $x_{1} \neq x_{2}$. Given that variables $x_{1}, \ldots, x_{n}$ must all be pairwise different, a constraint of the type all_different $\left(x_{1}, \ldots, x_{n}\right)$ can be used to formulate in a compact manner the $\frac{n(n-1)}{2}$ binary constraints of difference. Such an $n$-ary constraint is also called a predicate because it imposes a logical condition on its variable set. Constraint Programming $(C P)$ makes use of such elaborate predicates in order to provide a natural modelling framework ([2]). Such models are solved by CP techniques designed to produce feasible solutions. Alternatively, Integer Programming (IP) methods can be employed in cases where a logic predicate can be represented by linear inequalities involving integer variables ([1]). Apparently, such representations are important not only because they enrich the arsenal of resolution techniques but also because they motivate the integration of CP and IP in a unified modelling and algorithmic framework (see [3]).

An efficient representation of a predicate must be tight, i.e. it must include facet-defining inequalities of the convex hull of integer solutions satisfying the predicate. Such representations have been proposed for the all_different predicate ([8]), for cardinality rules ([6]) and for the sum constraint ([7]). A next step would be to derive such representations for sets of more that one predicates. The current paper works towards this direction by studying a system of two all_different constraints which may share a number of variables. In particular, we examine the polytope defined by the convex hull of integer vectors satisfying the system of the two all_different predicates. The dimension of this polytope is established and subsequently two classes of facet-defining inequalities are exhibited. These classes are of exponential size, a fact that

\footnotetext{
${ }^{*}$ Corresponding address: D. MAGOS, 30 Theodorou Geometrou Str., Athens 11743, Greece. Email:dmagos@teiath.gr
} 


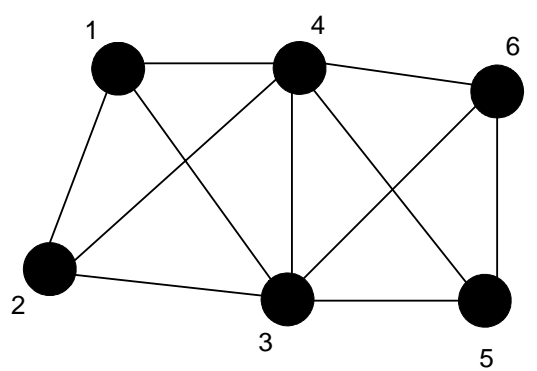

Figure 1: A graph-coloring example

prohibits their explicit use in a Linear Programming (LP) model. We resolve this difficulty by introducing a separation algorithm of low complexity, which provides only the facet-defining inequalities violated by a given vector. We also note that all these results can be directly applied to the optimization problem involving the (min-)maximization of a linear function over the system of the two all_different predicates.

\section{Mathematical formulation and applications}

The system consists of two all_different predicates, each including $n$ variables. Let $J_{1}\left(J_{2}\right)$ denote the set indexing the variables of the first (second) predicate, where $\left|J_{1}\right|=\left|J_{2}\right|=n$. Let also $D(D \subset \mathbb{Z})$ denote the domain of each variable with $|D|=k$. For the system to be feasible, it must be that $k \geq n$. For simplicity, assume $D=\{0,1, \ldots, k-1\}$. The system can be written as follows.

$$
\begin{aligned}
& \text { all_different }\left\{x_{j}: j \in J_{1}\right\}, \\
& \text { all_different }\left\{x_{j}: j \in J_{2}\right\}, \\
& x_{j} \in D, \forall j \in J_{1} \cup J_{2}
\end{aligned}
$$

Let $T$ denote the (possibly empty) subset of indices of the variables appearing in both predicates. In short, $T=J_{1} \cap J_{2}$ with $|T|=t$. We denote as $I_{p}=J_{p} \backslash T$, for $p=1,2$, i.e. $I_{p}$ is the index set of the non-common variables of each predicate. Let us provide two examples.

Example 1 (Graph coloring [4]) Consider the graph of Figure 1. We wish to color each node in such a way that the endpoints of every edge are assigned a different color. For simplicity, assume that there are four colors available for every node, namely $D=\{$ red, blue, green, orange $\}$. Let $x_{i}$ denote the color used for node $i$, with $D_{i}=D$, for $i=1, \ldots, 6$. Observe that this graph is formed by two cliques that have two nodes in common. Clearly, the colors assigned to the nodes of each clique must be pairwise different. Therefore, the 
coloring problem for this graph can be modelled via two all_different predicates:

$$
\begin{aligned}
& \text { all_different }\left\{x_{1}, x_{2}, x_{3}, x_{4}\right\}, \\
& \text { all_different }\left\{x_{3}, x_{4}, x_{5}, x_{6}\right\}
\end{aligned}
$$

According to our notation, $J_{1}=\{1,2,3,4\}, J_{2}=\{3,4,5,6\}, T=\{3,4\}$.

Example 2 (Timetabling) Consider a two-day course, where students are allocated into five groups $\{a, b, c, d, e\}$. Every group is assigned to a single teacher on each day of the course. To minimise the effort for teachers working on both days, the timetable should assign to them a single group throughout the course. Let the teachers available on the first and second day of the course be $\{1,2,3,4,5\}$ and $\{3,4,5,6,7\}$, respectively, i.e. teachers $\{3,4,5\}$ are teaching on both days. If $x_{i}$ denotes the group tutored by teacher $i$, with $D_{i}=\{a, b, c, d, e\}$, the timetabling problem for this course is modelled as follows:

$$
\begin{aligned}
& \text { all_different }\left\{x_{1}, x_{2}, x_{3}, x_{4}, x_{5}\right\}, \\
& \text { all_different }\left\{x_{3}, x_{4}, x_{5}, x_{6}, x_{7}\right\}
\end{aligned}
$$

Again, our notation implies that $J_{1}=\{1,2,3,4,5\}, T=\{3,4,5\}$ and $I_{1}=\{1,2\}$.

The convex hull of integer solutions to the system (1), (2) is denoted as $P_{I}$, i.e.

$$
P_{I}=\operatorname{conv}\left\{x \in D^{2 n-t}:(1),(2) \text { are satisfied }\right\}
$$

Let $P_{L}$ denote a linear programming (LP) relaxation of $P_{I}$. For $k>n$, we consider $P_{L}$ to be

$$
k-1 \geq x_{j} \geq 0, \forall j \in J_{1} \cup J_{2}
$$

whereas if $k=n, P_{L}$ is described by

$$
\begin{aligned}
& \sum\left\{x_{j}: j \in J_{1}\right\}=\frac{n(n-1)}{2}, \\
& \sum\left\{x_{j}: j \in I_{2}\right\}-\sum\left\{x_{j}: j \in I_{1}\right\}=0, \\
& x_{j} \geq 0, \forall j \in J_{1} \cup J_{2}
\end{aligned}
$$

To facilitate the discussion of the following section, we adopt some conventions. For a matrix $X$, let $X(i, j)$ denote the element appearing at row $i$ and column $j$. Let \# $\operatorname{col}(X)(\# \operatorname{row}(X))$ denote the number of columns (rows) of $X$. Assume that $Y$ is another matrix such that $\# \operatorname{col}(Y) \leq \# \operatorname{col}(X)$ and $\# \operatorname{row}(Y) \leq \# \operatorname{row}(X)$. 
For conciseness, we introduce the operation $Y \leftarrow X$ to imply the assignment of $X(i, j)$ to $Y(i, j)$, where indices $i$ and $j$ span only the rows and columns of matrix $Y$.

\section{Facets of $P_{I}$}

We commence the polyhedral analysis of $P_{I}$ by establishing its dimension. By definition, $P_{I} \subset P_{L}$, therefore $\operatorname{dim} P_{I} \leq \operatorname{dim} P_{L}$. We will prove that $\operatorname{dim} P_{I}=\operatorname{dim} P_{L}$ by exhibiting $\operatorname{dim} P_{L}+1$ affinely independent vectors of $P_{I}$. First observe that, for $k>n, P_{L}$ is full-dimensional, whereas for $k=n P_{L}$ is defined in terms the equality constraints (3), (4), which form a system of full row rank.

\section{Theorem 3}

$$
\operatorname{dim} P_{I}= \begin{cases}2 n-t, & \text { if } k>n, \\ 2(n-1)-t, & \text { if } k=n\end{cases}
$$

Proof. Let $T=\{n-t+1, \ldots, n\}, I_{1}=J_{1} \backslash T=\{1, \ldots, n-t\}, I_{2}=J_{2} \backslash T=\{n+1, \ldots, 2 n-t\}$. Consider a matrix $B$, where each row defines an integer point of $P_{I}$ and each column is associated with a specific variable. Thus, entry $B(i, j)$ is the value of variable $x_{j}$ at point $i \in P_{I}$. For $k=n$, we consider $\# \operatorname{row}(B)=2 n-t-1$, whereas for $k>n \# \operatorname{row}(B)=2 n-t$.

Let the first $n-t$ columns correspond to variables $x_{1}, \ldots, x_{n-t}$, the next $t$ columns to variables $x_{n-t+1}, \ldots, x_{n}$ and the last $n-t$ columns to variables $x_{n+1}, \ldots, x_{2 n-t}$. Hence, the sets of indices $I_{1}, T, I_{2}$ partition the columns of $B$ into three sets. By also splitting the rows of $B$ into two sets, we impose the following partitioning of $B$ into six submatrices:

$$
B=\left[\begin{array}{ccc}
C_{I_{1}} & C_{T} & C_{I_{2}} \\
D_{I_{1}} & D_{T} & D_{I_{2}}
\end{array}\right]
$$

Clearly, $\# \operatorname{col}\left(C_{I_{1}}\right)=\# \operatorname{col}\left(D_{I_{1}}\right)=n-t, \# \operatorname{col}\left(C_{T}\right)=\# \operatorname{col}\left(D_{T}\right)=t$ and $\# \operatorname{col}\left(C_{I_{2}}\right)=\# \operatorname{col}\left(D_{I_{2}}\right)=$ $n-t$. To illustrate the contents of $B$, we initially examine the first three submatrices. Matrices $C_{I_{1}}, C_{T}$ and $C_{I_{2}}$ include $n$ rows. The submatrix formed by $C_{I_{1}}$ and $C_{T}$ contains all cyclic permutations of elements $\{n-1,0,1, \ldots, n-2\}$. It is illustrated next (the vertical line separates the entries of $C_{I_{1}}$ from these of $C_{T}$ )

Also $C_{I_{2}} \leftarrow C_{I_{1}}$, i.e. $C_{I_{2}}$ is identical to $C_{I_{1}}$.

$$
\left[C_{I_{1}} \mid C_{T}\right]=\left[\begin{array}{cccccc|ccc}
\mathrm{n}-1 & 0 & \cdots & \mathrm{n}-\mathrm{t}-4 & \mathrm{n}-\mathrm{t}-3 & \mathrm{n}-\mathrm{t}-2 & \mathrm{n}-\mathrm{t}-1 & \cdots & \mathrm{n}-2 \\
\mathrm{n}-2 & \mathrm{n}-1 & \cdots & \mathrm{n}-\mathrm{t}-5 & \mathrm{n}-\mathrm{t}-4 & \mathrm{n}-\mathrm{t}-3 & \mathrm{n}-\mathrm{t}-2 & \cdots & \mathrm{n}-3 \\
\vdots & \vdots & \vdots & \vdots & \vdots & \vdots & \vdots & \vdots & \vdots \\
0 & 1 & \cdots & \mathrm{n}-\mathrm{t}-3 & \mathrm{n}-\mathrm{t}-2 & \mathrm{n}-\mathrm{t}-1 & \mathrm{n}-\mathrm{t} & \cdots & \mathrm{n}-1
\end{array}\right]
$$

The number of rows for $D_{I_{1}}, D_{T}, D_{I_{2}}$ depends on $k$ : for $k=n$ it equals $n-t-1$, whereas for $k>n$ it equals $n-t$. These two cases are examined separately in our proof. We set $D_{I_{1}} \leftarrow C_{I_{1}}$ to emphasise that matrix $D_{I_{1}}$ receives only the first \# $\operatorname{row}\left(D_{I_{1}}\right)$ rows of matrix $C_{I_{1}}$. For example, in the case of $k=n$, the 
last $t+1$ rows of $C_{I_{1}}$ are not contained in $D_{I_{1}}$.

In an analogous manner, we set $D_{T} \leftarrow C_{T}$.

$$
D_{I_{1}}=\left[\begin{array}{cccccc}
\mathrm{n}-1 & 0 & \cdots & \mathrm{n}-\mathrm{t}-4 & \mathrm{n}-\mathrm{t}-3 & \mathrm{n}-\mathrm{t}-2 \\
\mathrm{n}-2 & \mathrm{n}-1 & \cdots & \mathrm{n}-\mathrm{t}-5 & \mathrm{n}-\mathrm{t}-4 & \mathrm{n}-\mathrm{t}-3 \\
\vdots & \vdots & \vdots & \vdots & \vdots & \vdots \\
\mathrm{t}+1 & \mathrm{t}+2 & \cdots & \cdots & \cdots & \cdots
\end{array}\right]
$$

To better illustrate the construction of submatrix $D_{I_{2}}$, it is convenient to consider an alternative partitioning of $B$ :

$$
B=\left[\begin{array}{ccc}
C_{I_{1}, T} & \bar{C}_{I_{2}} & p \\
D_{I_{1}, T} & \bar{D}_{I_{2}} & q
\end{array}\right]
$$

where $C_{I_{1}, T}=\left[C_{I_{1}} \mid C_{T}\right], D_{I_{1}, T}=\left[D_{I_{1}} \mid D_{T}\right], C_{I_{2}}=\left[\bar{C}_{I_{2}} \mid p\right], D_{I_{2}}=\left[\bar{D}_{I_{2}} \mid q\right]$ with $p, q$ being column vectors. The actual contents of $\bar{D}_{I_{2}}$ and $q$ are analysed within the following cases.

Case $1 k=n$

Matrix $D_{I_{1}}$ includes two top-left to bottom-right diagonals, namely $d_{1}, d_{2}$, of maximum size (i.e. $n-t-1$ ). Diagonals $d_{1}$ and $d_{2}$ include the values $B(n+r, r)$ and $B(n+r, r+1)$, respectively, for $r=1, \ldots, n-t-1$. We denote as $S$ the triangular part of $D_{I_{1}}$ including all the elements above $d_{1}$. Observe that diagonal $d_{2}$ is included in $S$, while diagonal $d_{1}$ is not. Thus, $S$ includes the elements $B(n+i, j)$, for $i=1, \ldots, n-t-1$, $j=i+1, \ldots, n-t$. Also let $Q$ denote the triangular part of $D_{I_{1}}$ including all the elements below $d_{1}$ (but not $d_{1}$ itself), i.e. $Q$ includes the elements $B(n+i, j)$, for $i=2, \ldots, n-t-1, j=1, \ldots, i-1$. Matrix $\bar{D}_{I_{2}}$ is identical to $D_{I_{1}}$ except from its diagonal $d_{1}$, which is omitted and instead placed in column vector $q$. Hence, matrix $\bar{D}_{I_{2}}$ contains $d_{2}$ as its single main diagonal. A schematic illustration of $D_{I_{1}}$ and $D_{I_{2}}$ is shown below.

$$
D_{I_{1}}=\left[\begin{array}{ccc}
\ddots & & S \\
& d_{1} & \\
Q & & \ddots
\end{array}\right], \quad D_{I_{2}}=\left[\begin{array}{cc|c}
\ddots & S & d_{1} \\
Q & \ddots &
\end{array}\right]
$$


As an example, we illustrate matrix $B$ for $n=6$ and $t=2$. Notice that the bordered elements belong to diagonal $d_{1}$ and appear also at the last column of $D_{I_{2}}$.

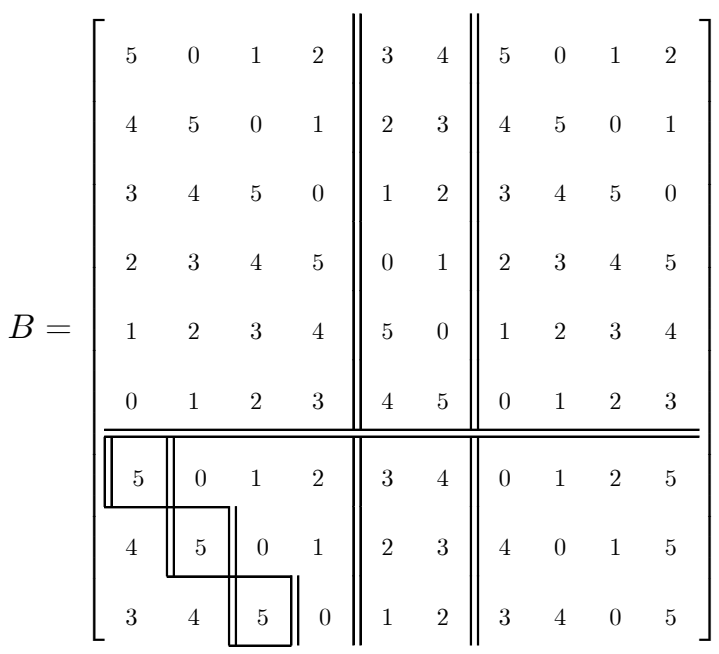

The following series of operations amount to subtracting the columns of $\left[C_{I_{1}} \mid D_{I_{1}}\right]^{T}$ from those of $\left[C_{I_{2}} \mid D_{I_{2}}\right]^{T}$. We set $O \leftarrow C_{I_{2}}-C_{I_{1}}$ and $E \leftarrow D_{I_{2}}-D_{I_{1}}$. Observe that $O$ is a matrix of zeros since $C_{I_{1}}=C_{I_{2}}$. By substituting in $B$ the submatrix $\left[C_{I_{2}} \mid D_{I_{2}}\right]^{T}$ by $[O \mid E]^{T}$, we obtain matrix $\bar{B}$. Evidently, $\operatorname{rank} B=\operatorname{rank} \bar{B}$. Let $E=[\bar{E} \mid \bar{q}]$ and $O=[\bar{O} \mid 0]$, where $\bar{q}$ is the last column of $E$ and 0 is a column vector of zeros. Analytically,

$$
\bar{B}=\left[\begin{array}{ccc}
C_{I_{1}, T} & \bar{O} & 0 \\
D_{I_{1}, T} & \bar{E} & \bar{q}
\end{array}\right]
$$

By construction, $\bar{E}$ is a square upper triangular matrix with non-zero elements in its main diagonal. To see this, notice that $Q$ appears in the same position both in $D_{I_{1}}$ and $D_{I_{2}}$ and $d_{1}, d_{2}$ differ in all elements. As a result, $\bar{E}(i, i)=d_{2}(i)-d_{1}(i) \neq 0$, for every $i$. Hence, $\bar{E}$ is non-singular. $C_{I_{1}, T}$ is a non-negative square cyclic matrix, therefore also non-singular. The determinant of the matrix obtained from $\bar{B}$ by deleting its last column (i.e. $[0 \mid \bar{q}]^{T}$ ) is equal to $\operatorname{det} C_{I_{1}, T} \cdot \operatorname{det} \bar{E} \neq 0$. Hence, the matrix $\bar{B}(B$ also) is of full row rank. Thus, the rows of $B$ illustrate $2 n-t-1$ linearly independent vectors of $P_{I}$.

\section{Case $2 k>n$}

For this case we must exhibit $2 n-t+1$ affinely independent points of $P_{I}$. Matrix $B$ remains as in the previous case except for a few changes. First, recall that submatrix $D_{I_{1}}$ (and also $D_{T}, D_{I_{2}}$ ) includes one additional row in this case. As a result, $D_{I_{1}}$ is now a square submatrix with a single diagonal of maximum size (i.e. $n-t)$, namely $d_{1}$. Moreover, the last entry of $d$ is set to $n$, i.e. $B(2 n-t, 2 n-t) \leftarrow n$. Consider the matrix

$$
B^{\prime}=\left[\begin{array}{l}
B \\
g
\end{array}\right]
$$


where row $g=(0,1, \ldots, n-2, n, 0, \ldots, n-t-1)$. It is sufficient to prove that the $2 n-t+1$ rows of $B^{\prime}$, each corresponding to an integer point of $P_{I}$, are affinely independent. Based on the definition of affine independence ([5]), this is equivalent to showing the non-singularity of the matrix

$$
M=\left[B^{\prime} \mid e\right]=\left[\begin{array}{c}
B \\
g
\end{array} \mid e\right]
$$

where $e(\# \operatorname{row}(e)=2 n-t+1)$ is a column vector of $1 \mathrm{~s}$. With respect to matrix $B^{\prime}$, we subtract the first $n-t$ columns from the last $n-t$ columns (i.e., perform the same elementary column operations as in the case of $k=n)$, thus obtaining the submatrix $[\bar{B} \mid \bar{g}]^{T}$, where $\bar{g}=(0,1, \ldots, n-2, n, 0, \ldots, 0)$. These operations transform the matrix $M$ to $\bar{M}$ which has the same rank as $M$. We consider the following partitioning of $\bar{M}$.

$$
\bar{M}=\left[\begin{array}{rrr}
C_{I_{1}, T} & O & e_{1} \\
D_{I_{1}, T} & E & e_{2} \\
\bar{g} & & 1
\end{array}\right]
$$

where $O, E$ are defined exactly as in the previous case and $e_{1}, e_{2}$ are column vectors of ones of appropriate size. Also observe that $O, E$ are square submatrices with $O$ containing zeros and $E$ being upper diagonal with non-zero entries in its leading diagonal.

To transform the last column of $\bar{M}$ into the vector $(0, \ldots, 0,1)^{T}$, we perform the following elementary operations: we subtract (a) the last row of $\left[C_{I_{1}, T}|O| e_{1}\right]$ from all other rows of this submatrix, and, (b) the row $[\bar{g} \mid 1]$ from the last row of $\left[C_{I_{1}, T}|O| e_{1}\right]$ and from the rows of $\left[D_{I_{1}, T}|E| e_{2}\right]$. The derived matrix, namely $M^{\prime}$, has the same rank as $\bar{M}$. Analytically,

$$
M^{\prime}=\left[\begin{array}{cc}
C_{I_{1}, T}^{\prime} & O^{\prime} \\
D_{I_{1}, T}^{\prime} & E^{\prime}
\end{array}\right] \text {, where } E^{\prime}=\left[\begin{array}{cc}
E & 0 \\
0^{T} & 1
\end{array}\right], C_{I_{1}, T}^{\prime}=\left[\begin{array}{cccccccc}
\mathrm{n}-1 & -1 & -1 & -1 & \cdots & -1 & -1 & -1 \\
\mathrm{n}-2 & \mathrm{n}-2 & -2 & -2 & \cdots & -2 & -2 & -2 \\
\mathrm{n}-3 & \mathrm{n}-3 & \mathrm{n}-3 & -3 & \cdots & -3 & -3 & -3 \\
\vdots & \vdots & \vdots & \vdots & \vdots & \vdots & \vdots & \vdots \\
2 & 2 & 2 & 2 & \cdots & 2 & -(\mathrm{n}-2) & -(\mathrm{n}-2) \\
1 & 1 & 1 & 1 & \cdots & 1 & 1 & -(\mathrm{n}-1) \\
0 & 0 & 0 & 0 & \cdots & 0 & 0 & -1
\end{array}\right]
$$

and $O^{\prime}=[O \mid 0]$ with 0 being a zero column vector.

It is easy to see that $E^{\prime}$ is non-singular. To show that $C_{I_{1}, T}^{\prime}$ is also non-singular, we perform operations in terms of rows and columns of $M^{\prime}$ that leave both $O^{\prime}$ and $E^{\prime}$ unaffected. First, we permute the columns of $C_{I_{1}, T}^{\prime}$ in such a way that its first column is shifted between columns $n-1$ and $n$. Then, to each row $j \in\{2, \ldots, n-1\}$, we add the multiple of the first row by $(n-j)$. The resulting matrix is upper diagonal with non zero elements in its leading diagonal. Hence, $\operatorname{det} M^{\prime}=\operatorname{det} C_{I_{1}, T}^{\prime} \cdot \operatorname{det} E^{\prime} \neq 0$ implying that $\bar{M}, M$ 
are non-singular.

The facets of the polytope defined as the convex hull of all vectors satisfying a single all_different constraint are given in [8]. For the system studied here, the corresponding classes of inequalities are

$$
\begin{aligned}
& \sum\left\{x_{s}: \quad s \in S\right\} \geq \frac{|S|(|S|-1)}{2}, \forall S \subseteq J_{p}, p=1,2, \\
& \sum\left\{x_{s}: \quad s \in S\right\} \leq \frac{|S|(2 k-|S|-1)}{2}, \forall S \subseteq J_{p}, p=1,2
\end{aligned}
$$

We note that if $k=n$ then $S \subseteq J_{p}$ is replaced by $S \subset J_{p}$ (strict inclusion). In this case, (5) and (6), taken for $S=J_{p}$, are satisfied as equalities by all points of $P_{I}$ therefore they cannot be facet-defining. For all other cases, we prove that (5), (6) induce facets of $P_{I}$.

We illustrate the result for inequality (5) and for $S \subset J_{2}$, all other cases of (5), (6) being symmetrical. Hence, for $H=\left\{i_{1}, i_{2}, \ldots, i_{h}\right\} \subset J_{2}$, consider the inequality

$$
x_{i_{1}}+x_{i_{2}}+\cdots+x_{i_{h}} \geq \frac{h(h-1)}{2}
$$

Define $F=\left\{x \in P_{I}: x_{i_{1}}+x_{i_{2}}+\cdots+x_{i_{h}}=\frac{h(h-1)}{2}\right\}$. Let $A$ denote the coefficient matrix of the minimum equality system for $P_{I}$. To prove that $(7)$ is facet-defining, we show that if there exists an equation $a x=a_{0}$ satisfied by all points of $F$, then $\left[a \mid a_{0}\right]$ can be written as a linear combination of the rows of $A$ and the coefficients of (7) (see [5]). Notice that, for $k=n$, the minimum equality system is defined by the linearly independent equalities (3), (4), while there exists no equality system for $k>n$. Therefore, $\operatorname{rank} A=2$ for $k=n$, while $\operatorname{rank} A=0$ for $k>n$. We examine these two cases separately.

Theorem 3 For $k=n, a \in \mathbb{R}^{2 n-t}, a_{0} \in \mathbb{R}$, if ay $=a_{0}$ holds for every $y \in F$, there exist scalars $\lambda_{1}, \lambda_{2}, \pi$ such that

$$
a_{i}=\left\{\begin{array}{c}
\lambda_{1}, i \in T \backslash H, \\
\lambda_{2}, i \in I_{2} \backslash H, \\
\lambda_{1}-\lambda_{2}, i \in I_{1}, \\
\lambda_{1}+\pi, i \in T \cap H, \\
\lambda_{2}+\pi, i \in I_{2} \cap H
\end{array}\right.
$$

and

$$
a_{0}=\lambda_{1} \frac{n(n-1)}{2}+\pi \frac{h(h-1)}{2}
$$

Proof. Evidently, there exists at least one $s \in\left(I_{2} \cup T\right) \backslash H$. If $s \in I_{2}$ define $\lambda_{1}=a_{1}+a_{s}, \lambda_{2}=a_{s}$, whereas if $s \in T$ define $\lambda_{1}=a_{s}, \lambda_{2}=a_{s}-a_{1}$. By substituting in (7), we obtain the coefficients of the $a$ 
Table 1: Values of $a_{i}$

\begin{tabular}{|l|l|l|}
\hline & $s \in I_{2}$ & $s \in T$ \\
\hline$i \in T \backslash H$ & $a_{1}+a_{s}$ & $a_{s}$ \\
\hline$i \in I_{2} \backslash H$ & $a_{s}$ & $a_{s}-a_{1}$ \\
\hline$i \in I_{1}$ & $a_{1}$ \\
\hline$i \in T \cap H$ & $a_{1}+a_{s}+\pi$ & $a_{s}+\pi$ \\
\hline$i \in I_{2} \cap H$ & $a_{s}+\pi$ & $a_{s}-a_{1}+\pi$ \\
\hline
\end{tabular}

Table 2: Proving (8) for $i \notin H$

\begin{tabular}{|l|l|l|}
\hline & $s \in I_{2}$ & $s \in T$ \\
\hline \multirow{2}{*}{$i \in T \backslash H$} & $x_{1}=1, x_{s}=1, x_{i}=0$, & $x_{i}=0, x_{s}=1$, \\
& $\bar{x}_{1}=0, \bar{x}_{s}=0, \bar{x}_{i}=1$ & $\bar{x}_{i}=1, \bar{x}_{s}=0$ \\
\hline \multirow{2}{*}{$i \in I_{2} \backslash H$} & $x_{s}=0, x_{i}=1$, & $x_{1}=1, x_{i}=1, x_{s}=0$, \\
& $\bar{x}_{i}=0, \bar{x}_{s}=1$ & $\bar{x}_{1}=0, \bar{x}_{i}=0, \bar{x}_{s}=1$ \\
\hline$i \in I_{1}$ & \multicolumn{2}{|c|}{$x_{1}=0, x_{i}=1, \bar{x}_{1}=1, \bar{x}_{i}=0$} \\
\hline
\end{tabular}

vector $\left(a_{i}, \forall i \in J_{1} \cup J_{2}\right)$ illustrated in Table 1 . We must prove that each $a_{i}$ is equal to the value depicted in the corresponding cell of the table. We briefly describe the methodology followed. For each case, we consider two integer points $x, \bar{x} \in F$. By hypothesis, both points satisfy $a y=a_{0}$, therefore equation $a x=a \bar{x}$ holds. By properly selecting $x$ and $\bar{x}$, the desired result is obtained after cancelling identical terms in equation $a x=a \bar{x}$.

We illustrate analytically this approach for $i \in T \backslash H$, and for $s \in I_{2}$. Assume an integer point $x \in F$ such that $x_{1}=1, x_{s}=1, x_{i}=0$. Notice that this point satisfies both all_different constraints and also (7) as an equality. By hypothesis, it holds that $a x=a_{0}$ or analytically:

$$
a_{1}+a_{s}+0 \cdot a_{i}+\sum\left\{a_{j} x_{j}: j \in\left(I_{1} \cup I_{2} \cup T\right) \backslash\{1, s, i\}\right\}=a_{0}
$$

Also consider the integer point $\bar{x}$, such that $\bar{x}_{1}=0, \bar{x}_{s}=0, \bar{x}_{i}=1, \bar{x}_{j}=x_{j}$ for all $j \in\left(I_{1} \cup I_{2} \cup T\right) \backslash\{1, s, i\}$. Observe that $\bar{x} \in F$. We can write equation $a \bar{x}=a_{0}$ in the following form:

$$
0 \cdot a_{1}+0 \cdot a_{s}+a_{i}+\sum\left\{a_{j} x_{j}: j \in\left(I_{1} \cup I_{2} \cup T\right) \backslash\{1, s, i\}\right\}=a_{0}
$$

It is easy to see that equations (10) and (11) imply $a_{i}=a_{1}+a_{s}$, as required.

The remaining cases for $i \notin H$ can be checked in the same fashion through Table 2 . This table depicts only the relevant values of $x, \bar{x} \in F$; the remaining ones have identical values in both $x$ and $\bar{x}$, thus resulting in the corresponding terms of $a x=a \bar{x}$ to cancel out. 
It remains to prove our claim for $i \in H$. For $s \in I_{2}$, define

$$
\pi_{i}= \begin{cases}a_{i}-a_{1}-a_{s}, & \text { for } i \in T \cap H \\ a_{i}-a_{s}, & \text { for } i \in I_{2} \cap H\end{cases}
$$

We will show that all $\pi_{i}$ are equal. Let $i_{s}, i_{q} \in T \cap H$. Consider an integer point $x \in F$ with $x_{i_{q}}=1, x_{i_{s}}=0$ and $\bar{x}$ having $\bar{x}_{i_{s}}=1, \bar{x}_{i_{q}}=0, \bar{x}_{m}=x_{m}$, for all $m \in\left(J_{1} \cup J_{2}\right) \backslash\left\{i_{s}, i_{q}\right\}$. Then, equation $a x=a \bar{x}$, after cancelling identical terms and substituting the remaining terms from (12), yields $\pi_{i_{s}}=\pi_{i_{q}}$. A similar result is valid for $i_{s}, i_{q} \in I_{2} \cap H$. Finally, let $i_{q} \in T \cap H$ and $i_{s} \in I_{2} \cap H$. Consider an integer point $x \in F$ with $x_{i_{q}}=1, x_{1}=x_{i_{s}}=0$ and $\bar{x}$ having $\bar{x}_{1}=\bar{x}_{i_{s}}=1, \bar{x}_{i_{q}}=0, \bar{x}_{m}=x_{m}$, for all $m \in\left(J_{1} \cup J_{2}\right) \backslash\left\{1, i_{s}, i_{q}\right\}$. Thus, $a x=a \bar{x}$ yields $a_{i_{q}}=a_{i_{s}}+a_{1}$. By substituting terms $a_{i_{q}}, a_{i_{s}}$ from (12) and cancelling out identical terms, we obtain $\pi_{i_{s}}=\pi_{i_{q}}=\pi$.

For $s \in T$, define

$$
\pi_{i}= \begin{cases}a_{i}+a_{1}-a_{s}, & \text { for } i \in I_{2} \cap H, \\ a_{i}-a_{s}, & \text { for } i \in T \cap H\end{cases}
$$

The proof is carried out in a manner analogous to that of the previous case.

The proof of (8) is complete. To show (9), consider $s \in I_{2}$ and an arbitrary integer point $x \in F$. Then $a x=a_{0}$ can be written as

$$
a_{0}=\sum_{i \in I_{1}} a_{i} x_{i}+\sum_{i \in\left(I_{2} \cup T\right) \backslash H} a_{i} x_{i}+\sum_{i \in H} a_{i} x_{i}
$$

By substituting all terms from Table 1, we obtain

$$
\begin{aligned}
a_{0}= & a_{1} \sum_{i \in I_{1}} x_{i}+a_{s} \sum_{i \in I_{2} \backslash H} x_{i}+\left(a_{1}+a_{s}\right) \sum_{i \in T \backslash H} x_{i} \\
& +\left(a_{s}+\pi\right) \sum_{i \in I_{2} \cap H} x_{i}+\left(a_{1}+a_{s}+\pi\right) \sum_{i \in T \cap H} x_{i} \\
= & a_{1} \sum_{i \in I_{1} \cup T} x_{i}+a_{s} \sum_{i \in I_{2} \cup T} x_{i}+\pi \sum_{i \in H} x_{i} \\
= & \left(a_{1}+a_{s}\right) \frac{n(n-1)}{2}+\pi \frac{h(h-1)}{2}
\end{aligned}
$$

In an analogous manner we show (9) for $s \in T$.

Establishing an analogous result for $k>n$ is simpler.

Theorem 4 For $k>n, a \in \mathbb{R}^{2 n-t}, a_{0} \in \mathbb{R}$, if ay $=a_{0}$ holds for every $y \in F$, there exists a scalar $\pi$ such that

$$
a_{i}=\left\{\begin{array}{l}
0, i \in\left(J_{1} \cup J_{2}\right) \backslash H, \\
\pi, i \in H
\end{array}\right.
$$


and

$$
a_{0}=\pi \frac{h(h-1)}{2}
$$

Proof. As in the previous proof, we define $\pi_{i}=a_{i}$, for all $i \in H$, and prove that all $\pi_{i}$ are equal. Let $i_{s}, i_{q} \in H$. Since $H \subset J_{2}$, we must examine the following cases: (i) $i_{s}, i_{q} \in I_{2} \cap H$, (ii) $i_{s}, i_{q} \in T \cap H$ and (iii) $i_{s} \in I_{2} \cap H, i_{q} \in T \cap H$. For the first two cases, the proof proceeds exactly as in Theorem 3 . For the third case, consider an integer point $x \in F$ such that $x_{i_{q}}=1, x_{i_{s}}=0$ and $x_{i} \neq 1$, for all $i \in I_{1}$. Such a point exists only for $k>n$, since there are enough values in $D \backslash\{1\}$ to be assigned to the variables indexed by $I_{1}$. Consider also $\bar{x} \in F$ such that $\bar{x}_{i_{q}}=0, \bar{x}_{i_{s}}=1, \bar{x}_{i}=x_{i}$, for all $i \in\left(J_{1} \cup J_{2}\right) \backslash\left\{i_{s}, i_{q}\right\}$. Equation $a x=a \bar{x}$ yields $\pi_{i_{q}}=\pi_{i_{s}}=\pi$.

To show (14), consider an arbitrary integer point $x \in F$. Then,

$$
a x=\sum_{i \in H} a_{i} x_{i}+\sum_{i \in\left(J_{1} \cup J_{2}\right) \backslash H} a_{i} x_{i}=\pi \cdot \sum_{i \in H} x_{i}+0 \cdot \sum_{i \in\left(J_{1} \cup J_{2}\right) \backslash H} x_{i}=\pi \frac{h(h-1)}{2}
$$

The above theorems imply the following.

Corollary 5 For $n \geq 2$, inequalities (5) and (6) define facets of $P_{I}$.

\section{A separation algorithm}

Linear programming can be employed to provide a point of $P_{L}$. Checking whether this solution violates the facet-defining inequalities (5), (6) constitutes the separation problem. Separation is important because violated inequalities can be added to the linear program, thus obtaining a tighter LP relaxation. In our case, solving the separation problem by a brute-force method is not efficient since the number of inequalities is exponential in $n$, i.e. equals $2\left(2^{n}-1\right)$. Next, we present a polynomial-time separation algorithm which, given $x \in P_{L}$, either ends up with a violated inequality belonging to (5), (6) or proves that no such inequality exists. Comments are included in $/ * * /$.

Algorithm $6 /{ }^{*}$ Input $x \in P_{L}^{*} /$

Step 1:v $\leftarrow 0, u \leftarrow 0$;

Step 2: Sort the variable of $x$ in ascending order, in terms of their values, deriving $\left\{x_{i_{1}}, x_{i_{2}}, \ldots, x_{i_{n}}\right\}$;

Step3:For $h=1, \ldots, n$,

\{

$v \leftarrow v+x_{i_{h}} ;$

if $v<\frac{h(h-1)}{2}$ then return; ( ${ }^{*}$ Inequality $\sum_{j=1}^{h} x_{i_{j}} \geq \frac{h(h-1)}{2}$ is violated $^{*} /$ )

$u \leftarrow u+x_{i_{n+1-h}} ;$

if $u>\frac{h(2 k-h-1)}{2}$ then return; (/* Inequality $\sum_{j=1}^{h} x_{i_{n+1-j}} \leq \frac{h(2 k-h-1)}{2}$ is violated vi) $^{*}$

\}

Proposition 7 Algorithm 6 determines in $O\left(n \log _{2} n\right)$ steps whether a facet of $P_{I}$ described by (5) (6) is violated. 
Proof. Because of the ordering it holds that $\sum_{j=1}^{h} x_{i_{j}} \leq \sum\left\{x_{s}: s \in S\right\}$ for every $S \subseteq J_{p},|S|=h$. Hence if there exists $S(|S|=h)$ such that $\sum\left\{x_{s}: s \in S\right\}<\frac{h(h-1)}{2}$ then $\sum_{j=1}^{h} x_{i_{j}}<\frac{h(h-1)}{2}$. The case is similar for the inequalities (6). The complexity of Step 3 is $O(n)$. Thus, the most expensive operation is the sorting of the variables (Step 2), which can be accomplished easily in $O\left(n \log _{2} n\right)$ time.

\section{References}

[1] K. Darby-Dowman, J. Little, Properties of some combinatorial optimization problems and their effect in the performance of integer programming and constraint logic programming, INFORMS J. Comput. 10 (1998) 276-286.

[2] P. van Hentenryck, Constraint Satisfaction in Logic Programming, MIT Press, Boston, MA, 1989.

[3] J. N. Hooker, Logic Based Methods for Optimization, Wiley-Inter-Science, New York, NY, 2000.

[4] C. Le Pape, Private communication, ILOG S.A., 2004.

[5] W. R. Pulleyblank, Polyhedral combinatorics, in: G. L. Nemhauser, A. H. G. Rinnooy Kan, M. J. Todd, (Eds.) Optimization, North-Holland, Amsterdam, 1989, pp. 371-446.

[6] H. Yan, J. N. Hooker, Tight Representation of Logic Constraints as Cardinality Rules, Math. Program. 85 (1999) 363-377.

[7] T. H. Yunes, On the sum constraint: relaxation and applications, in: P. van Hentenryck, (Ed.) Principles and Practice of Constraint Programming - CP2002, LNCS 2470, Springer, Berlin, 2002, pp. 80-92.

[8] H. P. Williams, H. Yan, Representations of the all_different predicate of constraint satisfaction in integer programming, INFORMS J. Comput. 13 (2001) 96-103. 